\title{
LA JUSTIFICACIÓN DE LA LIBERTAD DEL HOMBRE FRENTE A LA OMNIPOTENCIA DIVINA EN LA ÉTICA DE GUILLERMO DE OCKHAM
}

\author{
Olga L. Larre \\ CONICET (República Argentina)
}

\section{RESUMEN}

El tema de la libertad no ha sido considerado sistemáticamente por Ockham; por ello la autora lo analiza desde la circunscripción de los presupuestos metodológicos transitados por el Venerabilis Inceptor para llegar a su elaboración. Se fundamenta, en un primer momento, la nueva justificación gnoseo-ontológica de la realidad propuesta en el ockhamismo. Ello permite definir los límites en los cuales se inscribe el tema según un modelo fuertemente inspirado en el principio de la omnipotencia divina.

La exposición ockhamista utiliza el lenguaje y la doctrina aristotélica del libre arbitrio, pero desde una semántica completamente diversa; pues la libre elección aristotélica se produce a partir de un primer reconocimiento de opciones entre las que el agente elige; de allí que la libertad resulte una función del intelecto.

Ockham desestima este elemento fundamental del aristotelismo al invertir la perspectiva del análisis; sostiene que la voluntad es un poder activo cuya opción fundamental - actuar o no actuar- no depende del intelecto sino de ella misma como causa sui.

La metafisica y la antropología que sostienen esta concepción han sido modificadas sustancialmente respecto de las posiciones dominantes del siglo XIII: la naturaleza carece de una orientación específica; no está abierta, ni determinada por la verdad ni por el bien. Sólo el agente libre y consciente actúa en vista de un fin que él mismo se impone, mientras que el universo físico es predecible y perfectamente determinado.

La libertad aparece como un comienzo frente a la determinación natural, configurándose, así, la primera versión medieval del dilema moderno de la libertad versus el determinismo.

Palabras clave: Ockham, Libertad, Omnipotencia, Autodeterminación, Determinismo.

\begin{abstract}
The freedom issue has not been systematically considered by Ockham; that is why the author approaches it from the point of view of the methodological principles examined by the Venerabilis Inceptor in order to reach its development. First, the basis for the new gnoseo-ontological justification of the reality proposed by the Ockham's followers is established. That makes it possible to set the limits in which the issue is framed according to a model strongly inspired in the principle of divine omnipotence.

Ockham's approach uses the Aristotelian language and doctrine of free will, but from a completely different point of view; since the Aristotelian free will results from a first recognition of a number of possibilities from which the agent opt; then freedom is an intellectual capability.

Ockham sets aside this key element of Aristotelianism by changing the approach of study; he supports that will is an active power and its key option - acting or not acting - does not depend on the human intellect but on itself as a causa sui.

Metaphysic and Anthropology, which support this belief, have been materially changed as compared to the main trends of the XIII Century: nature lacks a specific direction; it is not determined by truth and good. Only the free and conscious agent acts towards and end established by himself while the physical universe is predictable and perfectly determined.
\end{abstract}


Freedom is shown as a new reality against natural determination, thus shaping the first medieval version for the modern dilemma of freedom versus determinism.

Key words: Ockham, Freedom, Omnipotence, Self-determination, Determinism.

La rehabilitación del pensamiento medieval, dentro de los límites de la experiencia histórica nos permite considerar la evolución de la civilización occidental como una perfecta continuidad. Es en el medioevo, y más precisamente, entre 1250 y 1350 el tiempo en el que han aparecido las premisas de la situación intelectual contemporánea, caracterizada tanto por una concepción unificada del conocimiento humano posible, como por el surgimiento del saber formalizado univoco que pretende reinar casi con exclusividad en nuestros días. En este contexto, la figura del fraile franciscano Guillermo de Ockham adquiere un relieve singular en cuanto artífice y portavoz de este profundo cambio.

La condena del aristotelismo en 1277 había creado un ambiente de reacción contra lo que se consideraba una aportación pagana: la naturaleza griega portadora de necesidad, a los ojos de los más ortodoxos parecía comprometer la incondicional libertad de la omnipotencia divina. En este ambiente de reacción cristiana frente al paganismo ha de situarse el contingentismo radical de Ockham, basado y formulado en su famoso principio de la omnipotencia divina.

El punto de partida de la obra filosófica de Ockham es hondamente teológico y queda concretado en el primer artículo del Credo cristiano: Credo in unum Deum Patrem Omnipotentem, enunciado con énfasis en la quaestio VI de las Quodlibeta, conforme al cual puede atribuirse a la potencia divina todo aquello que no incluya contradicción ${ }^{1}$.

En función de esta perspectiva teológica, el ya clásico estudio de $\mathrm{Ph}$. Boehner ${ }^{2}$ resume los principios fundamentales del ockhamismo en los siguientes puntos, tomados casi literalmente de la Quodlibeta $V I^{3}$ :

$1^{\circ}$ Todo es posible para Dios exceptuado aquello que incluya contradicción.

$2^{\circ}$ Todo lo que Dios produce a través de las causas secundarias puede producirlo y conservarlo inmediatamente y sin su ayuda.

$3^{\circ}$ Dios puede causar, producir y conservar algo — sea substancia o accidente- de un modo separado respecto de cualquier otra realidad.

$4^{\circ}$ No se ha de afirmar que una proposición es verdadera o que una cosa existe, a menos que nos obligue la sola evidencia, la revelación, la experiencia, o la deducción lógica de una verdad revelada.

$5^{\circ}$ Toda realidad - exceptuado Dios- es contingente en cuanto a su ser.

Como consecuencia de esta polarización hacia la omnipotencia divina, Ockham da un sentido radical a la contingencia de todo lo creado. Si hay un orden en la estructura de lo real, será, en definitiva, también contingente. Y este orden ha de incluir la actividad de las «naturalezas», reuniendo la multitud dispersa de los singulares no en compartimentos de nivel necesario metafísico, sino configurando lo que él llamará la Potentia Dei Ordinata, orden contingente frente a lo que será la piedra de toque metafísica del ockhamismo: la Potentia Dei Absoluta.

Esta afirmación constituye, para Ockham, la expresión misma de la plena libertad del actuar y del querer divinos; pues fuera de la no-contradicción no hay ningún orden metafísico ${ }^{4}$, ni antes ni después de la afirmación de esta voluntad todopoderosa.

1 G. de Ockham, Quodlibeta VI, q. 6 (OTH IX, 604). Cfr. asimismo el comentario de Ph. Boehner, Ockham Philosophical writings, Nelson philosophical texts, Toronto, N. Y., Paris, 1959, Introducción, XIX-XXII.

2 Ph. Boehner, Ockham Philosophical Writings, Nelson philosophical texts, Toronto-New York, 1959, Introducción, XIX-XXII.

3 G. de Ockham, Quodlibeta VI, q. 6 (OTH IX, 604)

4 Cfr. la importancia concedida al tema por P. Vignaux, «Nominalisme», Dictionnaire de Théologie Catholique, Tome XI, 764-769. 
$\mathrm{Y}$ al indicar que Dios no puede hacer lo que es intrínsecamente imposible no se limita su poder; ya que carece de todo sentido hablar de lo intrínsecamente imposible. Quizá podría pensarse en un orden interior al mismo querer divino que consistiría en la ordenación de los medios al fin, una vez supuesta en Dios la elección y determinación de un orden concreto en el mundo. Así lo había entendido Escoto, pero Ockham no comulga con este psicologismo presente «in signo priori et posteriori» transferidos al interior de la vida divina ${ }^{5}$. Cierto que, una vez realizada la voluntad divina en la creación, la legalidad correspondiente al orden de la $\mathrm{Po}-$ tentia Dei Ordinata no deja de ser útil como medio de vinculación entre los singulares; pero, en realidad, la estructura metafísica de los individuos - únicos objetos de la creación- queda en el ockhamismo, fuera de toda uniformidad.

El énfasis dado al principio de la omnipotencia divina, convertido en regulador de toda su metafísica, trae como consecuencia el desarrollo de un radical sentido de la contingencia en el ámbito de lo real, junto con el establecimiento de nexos puramente accidentales entre los individuos. Es, asimismo, cierto que no puede probarse filosóficamente, pero una vez supuesto como artículo de fe, el mundo aparece con una luz especial. Todas las relaciones regulares se ven como contingentes en cuanto están sujetas a verificación experimental y no son materia de una deducción a priori; a ello se añade, además, que Dios podría producir algo sin la mediación de sus causas secundarias.

Desde luego, en todos los sistemas de pensamiento medieval la uniformidad y regularidad de los procesos naturales fue concebida de un modo accidental en cuanto sometida a la posible intervención de Dios por la vía del milagro. No obstante ello, la metafísica de las esencias le había conferido a la naturaleza una relativa estabilidad de la que Ockham la privó al reducir las relaciones y conexiones entre los entes, a la mera coexistencia, o bien, a la existencia sucesiva de «absolutos» ${ }^{6}$. A la luz de la omnipotencia divina, la contingencia de las relaciones y el orden de la naturaleza se vio como la expresión de la voluntad todopoderosa de Dios.

Este concepto ockhamista de naturaleza ha sido interpretado como una etapa en el camino hacia una concepción moderna de lo físico ${ }^{7}$; la vinculación es posible pero no suficiente pues, en mi opinión, no es conveniente concebir el trasfondo teológico como un mero agregado para Ockham. Su pensamiento tiene las características propias de la reflexión medieval, Dios constituye el núcleo de su investigación; con una clara exaltación de la omnipotencia y la libertad divina sobre cualquier otro atributo. Ockham como buen franciscano, es agustiniano, portavoz del Dios vivo de siempre; y tiene la clara aspiración de ser modesto testigo de una fe que busca conocer bien y explicar con sencillez.

Constituye nuestro propósito, en esta ocasión, revisar los pasos que Ockham transita para establecer su particular concepción de la libertad humana, condición y preámbulo para una justificación de la responsabilidad moral. El tema no ha sido analizado sistemáticamente por Ockham; por ello lo abordaremos desde la circunscripción de los presupuestos metodológicos transitados por el Venerabilis Inceptor para llegar a su elaboración.

Hemos de considerar, en un primer momento, la nueva fundamentación gnoseo-ontológica de la realidad propuesta en el ockhamismo. Esto nos permitirá definir los límites en los cuales se inscribe el tema de la libertad humana según un modelo fuertemente inspirado en la autosuficiente actividad divina.

5 Ockham, I Sent., q. 41 (OTH IV, 604)

6 El principio básico de la teoría de Ockham señala que sólo existen las res absolutae, mientras que todos los otros predicamentos se limitan a describir las condiciones del ente natural. Y admite dos clases de res absolutae: las substancias y las cualidades, más allá de las cuales nada es imaginable actual o potencialmente. De manera que sólo son objetivas las formas sensibles individuales, capaces cada una de ellas, de existencia independiente. He considerado el tema en: Olga L. Larre, La filosofia natural de Ockham. Una fenomenología del individuo, Eunsa, 2000,141 y ss.

7 Cfr. E. A. Moody, «Ockham and Aegidius of Rome», Franciscan Studies, 1949 (IX), 417-442. 


\section{LA PRESUPUESTOS EPISTEMOLÓGICOS DE UNA ONTOLOGÍA PRÓXIMA A LA FÍSICA}

La doctrina ockhamista del conocimiento se sitúa en un contexto de crítica del realismo ontológico en sus distintas formas, ya se trate del realismo absoluto de Duns Escoto o del moderado de Santo Tomás, sin llegar a comprender lo que distingue profundamente el segundo del primero.

Para los filósofos del siglo XIII la explicación inteligible de la realidad y la configuración racional de la inteligibilidad de esa realidad está condicionada por la afirmación de una naturaleza en la estructura óntica de los singulares.

Esta naturaleza, una vez descartadas las posiciones extremas del realismo exagerado de tipo platónico, vendrá a concretarse ya en la natura communis del escotismo, ya en la natura potentialis ofundamentum in re del realismo moderado. Por lo demás, esa natura ofrece la base de solución al eterno problema de los universales y es la garantía para la validez objetiva del conocimiento universal y necesario que constituye la ciencia. El objetivo de Ockham consiste, precisamente, en desentenderse de las naturalezas, al mismo tiempo que mantiene inquebrantable la afirmación de la posibilidad de una auténtica ciencia objetiva.

Ockham rechaza lo universal o la esencia, cualquiera sea el tipo de existencia que se le atribuya en la realidad. Los universales de Escoto son comprendidos por Ockham como realidades fisicas que constituyen la natura communis, con una unidad menor que la numérica y común a todos los individuos.

Su crítica recae, primero, sobre el tipo de unidad acordada por Escoto a la naturaleza, la que entiende ha de ser numéricamente una, o por el contrario, una colección de individuos. De este modo: es en lo real una pluralidad o, contrariamente, una unidad; pero no puede ser ambas sin caer en el equívoco 8 .

La crítica al realismo de Santo Tomás parte de la idea, también rechazada por Ockham, de que el universal es en potencia en el singular. Su visión del realismo moderado se halla filtrada por un tamiz de tendencia escotista: no olvidemos que este franciscano, antiescotista, pero formado en el escotismo de Oxford, sostiene una interpretación excesivamente «cosista» del universal y de la natura potencial que el realismo moderado pone sólo fundamentaliter in rebus ${ }^{9}$.

Frente a estas dos formas de realismo Ockham elabora una tesis personal que consiste en un rechazo de la teoria aristotélica de la abstracción donde la actividad de la inteligencia, es concebida de un modo semejante a la actividad sensitiva. El proceso de conocimiento se reduce así, a un contacto directo con la cosa, suprimiendo los intermediarios y concluyendo en una descripción de lo singular visto bajo diferentes aspectos.

En efecto, la gnoseología ockhamista aparece como la descripción de la manera conforme a la cual se efectúa el pasaje desde una pura aprehensión a la emisión de signos. El producto de este proceso son los términos y los conceptos, de cuyo estudio se ocupa la lógica; y que significan la cosa tal como nos es dada en la experiencia inmediata.

De este modo, el conocimiento intuitivo ejerce una función central en la epistemología ockhamista, pues conduce a la producción de dos conceptos en el espíritu: el concepto propio y el concepto de la especie a la cual éste pertenece. Por lo cual la intuición del singular es, a la vez, empírica e eidética: viendo a este hombre, veo un hombre. Precisamente un problema en este ámbito especulativo ockhamista radica en establecer cómo se produce el pasaje desde el conocimiento de la especie al del género, que no se realiza a través de una abstracción, en el sentido tradicional del término, sino mediante una ampliación del conocimiento intuitivo.

8 G. de Ockham, I Sent, d. 2, q. 6, (OTH II, 193).

9 G. de Ockham, Summa Logicae, I, cap. 17, (OPH I, 52). 
Por la intuición - relación inmediata entre el sujeto cognoscente y la cosa conocidatanto sensible como intelectual, se adquiere el conocimiento de cosas reales, no existiendo ningún ser que medie entre las cosas existentes y el espíritu que conoce. Para evitar la substancialización del concepto y la función de medio que puede desempeñar entre las cosas y el alma, Ockham utiliza a la intentio, como realidad en la mente y como simple tensión de la inteligencia. Así, la intentio singularis es la simple captación clara y distinta de lo real individual; y la intentio generalis, una captación inmediata e intuitiva, pero confusa, de ciertos rasgos comunes, semejantes, entre las cosas, que se da - como señalamos-al mismo tiempo que la intentio singularis. $\mathrm{Y}$ puesto que esta producción es cuasi-espontánea, parecen asimilarse en Ockham el orden conceptual con la realidad misma de la experiencia.

El concepto formado en la mente constituye un signo natural de la realidad misma, expresión de un estado de la inteligencia, que los hombres fijan a través de un lenguaje. Como es posible advertir, la noción peripatética de abstracción juega aquí una función limitada: el conocimiento abstractivo se aproxima a la capacidad de representar una cosa cuando no se encuentra actualmente presente, verificándose un verdadero repliegue del conocimiento abstractivo sobre el orden de la imaginación ${ }^{10}$.

Sobre la base de estos criterios, y en orden a la elaboración de la ciencia, Ockham no niega que puedan realizarse demostraciones propter quid -de causas a efectos-como las elaboradas a partir de principios ciertos e indemostrables; o deducciones de una verdad a partir de otras más universales, tal como se da en el silogismo. Pero señala que no es éste el único modo válido de hacer ciencia. Ockham propone proceder a través de un camino más sencillo: de lo simple a lo complejo, elevándose desde la intuición a la causa. Por esta vía podríamos encontrarnos con universales no justificados por una deducción propter quid. $\mathrm{Y}$, sin embargo, tenemos que concederles una validez significativa porque vienen dictados a partir de la experiencia. Así y por vía de ejemplo, no puede decirse que sea imposible alcanzar el conocimiento de la materia y de la forma de una cosa, partiendo del hecho que no se conozca su causa propter quid $^{11}$.

De este proceso, firmemente asentado por Ockham, se deducen importantes consecuencias. En primer lugar, el principio causal derivado a partir de la intuición no tiene por qué ser la causa propter quid de un hecho, sino sólo una causa hipotética. Por ello, en la elaboración de una ciencia, fácilmente nos podemos encontrar con diversas hipótesis para explicar una misma experiencia. Además, la búsqueda de las causas a partir de la experiencia no alcanza el pretendido rigor del paso de una forma a otra, o del conocimiento de una sustancia a su causa, sino que desde los accidentes, ciertamente dados en la intuición, sólo es posible sugerir causas hipotéticas. Con ello pierden consistencia, para Ockham, conceptos metafísicos tradicionales como el de substancia o el de causalidad, porque al tener conocimiento intuitivo sólo de los accidentes, la sustancia se convierte en un supuesto substrato, y la causalidad en una supuesta relación, quedando como base firme de conocimiento, solamente las intuiciones.

Ockham también se aparta de la tradición aristotélica en lo que concierne a la coordinación entre los sentidos y la inteligencia. El hombre en esta vida está sujeto al cuerpo y debe conocer las cosas sensiblemente, pero esto no significa que ambos modos de conocimiento estén coordinados. El conocimiento sensible se da a la par y sobre el mismo objeto que el conocimiento intelectual; $y$ en cierto modo lo condiciona, pero no es el material desde el cual el entendimiento producirá el universal. El conocimiento sensitivo y el intelectivo concluyen en

10 Una consideración sobre este problema podrá encontrarse en A. Ghisalberti, Guglielmo di Ockham, Vita e pensiero, Milano, 1972, 64-90.

11 G. de Ockham, Summula Philosophiae Naturalis, I, cap. 17 (OPH VI, 199-200). Hay traducción al castellano: G. de Ockham, Pequeña suma de Filosofia Natural, introducción, trad. y notas Olga L. Larre, Eunsa, 2003, 113. 
la cosa misma sin condicionarse mutuamente: la prueba de ello está en que un niño puede saber algo de manera sensitiva y no intelectivamente; y a la inversa, un alma separada -el alma de un bienaventurado- no conoce sensitiva sino intelectualmente ${ }^{12}$.

En síntesis: el concepto universal debe ser definido como un signo natural ${ }^{13}$ que se refiere a diversas realidades; es el componente elemental de las proposiciones y a él se subordina todo lenguaje oral y escrito ${ }^{14}$. La tesis presentada por Ockham es esencialmente semántica: une de manera implícita el ámbito especulativo que hoy estaría en manos de la psicología cognitiva, la teoría del signo (semiótica) y la teoría de la referencia. El universal no es una realidad sino un concepto. El concepto, a su vez, no es un simple contenido «objetivo» sino un acto que remite a una pluralidad de objetos singulares. Y este acto, finalmente, es un accidente real del espíritu, una cualidad del alma ${ }^{15}$, un acto cognitivo que no tiene existencia «objetiva» sino solamente un esse in anima por cuanto su existencia radica en ser una cualidad inherente en un sujeto $^{16}$.

Basándose en este nuevo fundamento epistemológico, Ockham reduce drásticamente la ontología; en cambio amplía el desarrollo de la física a la que consagró gran parte de su actividad intelectual e inviste a la lógica y a la metodología de una función doxolítica donde la pars destruens es a menudo más importante que su pars construens.

Es, precisamente, la lógica uno de los espacios intelectuales libres más importantes tras la condena de 1277 al que Ockham consagra gran parte de sus esfuerzos intelectuales. Desarrolla una tradición que había tenido importantes antecesores, alejada de la clásica comunión entre la lógica y una ontología ${ }^{17}$ que le impone sus reglas al razonamiento científico. De este modo, propone liberar al pensamiento de la confusión entre entidades lingüísticas y reales, entre los elementos del razonamiento y los elementos de la realidad.

En suma, Ockham afirma que no debemos atribuir a los signos - necesarios para describir y comunicar - ninguna otra función que la de ser señales indicativas de determinadas realidades, con lo cual la lógica adquiere un estatuto autónomo y formal, diverso de aquel que le concedieron sus predecesores de la via antiqua. Se opera, así, una separación entre lógica y realidad, entre términos y res, entre plano conceptual y real.

Con ello, Ockham enfatiza el rigor formal del razonamiento científico: la validez de una o de varias proposiciones se basa en el supuesto de que sujeto y predicado no significan cosas distintas entre sí, en un contexto equívoco, sino que indican la realidad designada. La fidelidad a la suposición lógica, en sus diversas formas, induce a eliminar expresiones ambiguas y a indicar en la realidad aquello de lo cual se habla. Se trata, en resumen, de un aparato lógico que establece un orden formal en el pensamiento, aclara el lenguaje y acentúa el camino de la experiencia centrado en la atención del conocimiento del individuo.

Como es posible advertir, y sintetizando ahora los elementos desarrollados, Ockham no duda del hecho del conocimiento científico humano; no se cuestiona el modo metafísico o psicológico de su adquisición. Lo que le preocupa es el recto uso del conocimiento y los límites de la acción de conocer. Parte del hecho mismo de conocer, para asentar una teoría que salva

12 Puede consultarse el problema en J. Biard, Logique et théorie du signe au quatorziène siècle, Paris, 1989 , 61.

13 El tema es extensamente tratado por T. de Andrés, El nominalismo de Guillermo de Ockham como filosofia del lenguaje, Gredos, 1969, 153-174.

14 P. Vignaux, «Nominalisme», Dictionnaire de Théologie Catholique, Tome XI, col. 750.

15 G. de Ockham, Summa Logicae, I, cap. 25 (OPH I, 75).

16 G. de Ockham, I Sent., d. 2, q. 8 (OTH II, 266 y ss.).

17 La lógica de Ockham ha adquirido en nuestro tiempo una singular relevancia, por su coincidencia temática con la lógica contemporánea. Cfr. El ya clásico estudio de Ph. Boelıner, Collected Articles on Ockham, St. Bonaventure, N.Y., 1958 174-200; 201-232; 232-267; 319-351. Otros estudios: A. De Muralt, L'enjeu de la Philosophie Médiévale, Brill, 1993. E. Moody, Studies in Medieval Philosophy, Science and Logic, Collected Papers 1933-1969, U. of California, 1975. 
todos los datos que se han de vincular. Es una metodología muy propia del siglo XIV: es probable una teoría que sea capaz de salvare phaenomena, entendiendo por tales todos los puntos constitutivos de un problema, según una entre las preselecciones posibles de un campo teórico. Ockham fuerza el carácter central del problema del conocer frente al del ser.

Si para Aristóteles la proposición: homo est homo es una proposición necesaria, y necesariamente verdadera; para Ockham, en cambio, esta proposición puede ser falsa, y lo sería desde el momento en que no existiese de hecho ningún hombre. La necesidad de esta proposición es hipotética y se expresa de esta manera: «si homo est, homo est homo». Es la existencia o la hipótesis de existencia de uno o varios singulares concretos lo que da -en opinión de Ockham-validez necesaria a las mismas definiciones metafísicas ${ }^{18}$.

\section{UNA ÉTICA FUNDADA EN LA INMANENCIA}

El agudo e innovador universitario aplica a los temas ético-políticos la misma epistemología y lógica que creó para la ciencia académica. En pocos casos la capacidad teórica pura y la acción práctica han andado tan hermanadas y en tan inquebrantable armonía.

El problema de abandonar el conocimiento de la substancia y las firmes relaciones causales, defendidas por la tradición aristotélica, le conducen a exponer una ética elaborada sobre hipótesis diversas respecto del siglo precedente. Los principios metodológicos que gobiernan su construcción son dos: en primer término, la fe constituye el recurso inapelable, una prueba más que nos advierte claramente la inserción de Ockham en el ámbito del pensamiento medieval; y luego, cuando la referencia a la fe no existe, el principio de economía impone su régimen $^{19}$.

La posición ética de Ockham se desarrolla sobre una antropología y una metafísica que ha exaltado el orden de la inmanencia: un cosmos sin finalidad, y una voluntad autónoma.

En efecto, el Venerabilis Inceptor considera inaceptable la posición de aquellos pensadores que, siguiendo a Aristóteles, conciben el mundo como un cosmos constituido por innumerables seres ordenados los unos a los otros conforme a una meta preestablecida. Su universo es, en cambio, una urdimbre de individuos, de res absolutae en movimiento. Y si bien sostiene que es necesario admitir una causa eficiente que conserve el universo en el ser ${ }^{20}$ es, en cambio, superflua la afirmación de la finalidad; pues el comportamiento de las cosas no conduce a admitir que obren en vistas de un fin.

En el mundo ockhamista de entes individuales contingentes, conocidos sólo proposicionalmente a partir de intuiciones atómicas, la relación entre dos entidades no penetra en su mismo ser para establecer un vínculo orgánico, como en la Grecia clásica, o en la metafísica del siglo XIII. La relación será para el maestro inglés una constatación extrínseca del espíritu que «se dice» el mundo. Así, en su sentido más literal, la causalidad eficiente no refleja un bonum diffusivun sui del acto, sino un hecio. «Causa es aquello por cuya existencia existe otra $\operatorname{cosa}{ }^{21}$, define Ockham múltiples veces con voluntad de evitar toda sugerencia de emanación, de conservación en el ser, de participación en 4 acto: una simple y pura sucesión de existencias que la mente constata como condición sin la cual no se da realmente la segunda entidad. En verdad la relación causal ockhamista es un post hoc, propter hoc específico. La necesidad

18 G. de Ockham, Summa Logicae, I, cap. 22 (OPH 65-66).

19 Sobre el tema: Olga L. Larre, "Ockham y una variante física del principio de economía», Analogía, 1997, $\mathrm{XI},(2), 165-182$.

20 El problema ha sido tratado en G. de Ockham, I Sent, 2, 10, (OTH, II, 338-350) y en las Quaestiones in libros Physicorum, QQ. 132-136 (OPH VI, 753-769).

21 G. de Ockham, Summa Logicae, I, 10 (OPH I, 37). 
y la universalidad se alcanzan, en el caso de la proposición que estáblece la relación causal, por el recurso a elementos formales. Si la causa eficiente queda así separada de una consideración ontológica, mucho más severa resulta ser la reducción operada sobre la causa final.

La causa final es precisamente causa en tanto hace que el agente actualice o produzca lo amado o deseado por él; de manera que, para que exista causalidad final se requieren dos condiciones: «que algo sea amado o deseado y que un agente produzca lo que ama o desea por sí mismo o en vistas de algo otro» ${ }^{22}$.

Este modo de actuar teleológico' es evidente en los seres que operan a proposito et sponte, pero no en aquellos inanimados: «me parece que es necesario afirmar que, según los principios de Aristóteles, los seres inanimados que actúan por necesidad natural no tendrían una causa final si no fueran movidos o dirigidos por alguien que conoce el fin» ${ }^{23}$. Pero, dado que esta causa final no es propiamente deseada por el agente natural sino por quien lo dirige o mueve, se la denomina sólo de modo impropio «causa final». Es este un giro muy corriente en Ockham cuando se propone negar una doctrina tradicionalmente admitida, adjudicarle un sentido impropio que acaba desvaneciéndose bajo el peso de su tesis central: no es posible admitir finalidad alguna en los seres inanimados. En efecto, mientras que la moción de la causalidad final en un sentido aristotélico connota la atracción ejercida por el fin sobre el agente; Ockham subordina la causalidad final a la eficiencia: «si el amor es causado por un fin amado visto como objeto, este fin no es una causa final sino eficiente ${ }^{24}$.

Su crítica se precisa en el Comentario a las Sentencias al indicar que la moción del fin no es, directamente real sino metafórica ${ }^{25}$. Y el mismo criterio aparece expresado en la Quodlibeta IV: «la causalidad del fin no es otra cosa que ser amado y deseado eficientemente por un agente ${ }^{26}$. Lo determinante, por tanto, no radica en la existencia o no del fin —que además siempre es fin para un sujeto- sino en la activa tendencia del sujeto hacia esa realidad.

En suma: según la definición expresada se puede hablar de finalidad sólo en agentes dotados de inteligencia y de voluntad y en una segunda acepción, impropia y amplia se entiende el fin, como el resultado de la acción de un agente que sigue el curso normal de la naturaleza, tal como si fuese previsto y deseado por ese agente.

Mas en el caso de estos agentes naturales conviene explicar la regularidad por el determinismo y no por la finalidad. Desde luego, si se presupone la existencia de Dios, se puede afirmar que las cosas inanimadas operan por fines determinados por Dios, Creador de sus naturalezas ${ }^{27}$, pero una proposición basada en la presuposición de la existencia de Dios no puede utilizarse, a su vez, para probar la existencia de otra. De este modo, Ockham transformó el orden cósmico en un orden en sí mismo indiferente, cuyo único valor radica en ser querido por Dios.

Con lo cual y en orden al tema que nos ocupa podemos extraer una primera conclusión: conocemos que las cosas tienden a un fin exclusivamente si Dios lo revela. Filosóficamente sólo arribamos a la conclusión que Dios puede ser el fin último de la creatura dotada de libertad y que el hombre puede no elegirlo. Por ello, sin la revelación el hombre ignoraría cuál es su fin último y no estaría en condiciones de establecer cuál es su verdadero bien.

En suma: para Ockham el orden moral no tiene un fundamento metafísico sino sólo teológico ${ }^{28}$.

G. de Ockham, Summula Philosophiae Naturalis, (OPH VI, 221).

G. de Ockham, Summula Philosophiae Naturalis, (OPH VI, 228).

Ockham, íb.

Ockham, II Sent., q. 5 (OTH V, 83).

Ockham, Quodl., IV, 1 (OTH IX, 293).

G. de Ockham, II Sent., 3 (OTH V, 50); Quod. 4, 1 (OTH IX, 295).

Cfr. M. Bastit, Les principes des choses en ontologie médiévale, Bière, 1997, 303 y ss. 
Una segunda transformación se opera, ahora, en el orden antropológico. Y así como la naturaleza ha quedado liberada de toda posible tensión o finalidad; los hombres, paralelamente, han sido liberados de toda necesaria tendencia hacia el bien o la verdad.

El acto humano escapa de las indicaciones dadas por el curso normal de las cosas y no se encuentra fundado sobre el orden de la naturaleza. Ockham cierra la acción humana a todo aquello que perturbe su autonomía, estableciendo que la decisión debe provenir del interior del espíritu. Suprime en el acto humano todo cuanto lo hace depender de lo exterior, y niega a la voluntad la capacidad de recibir y de acoger una indicación sobre lo que es bueno: «La operación interior es doble: una que inmediatamente está en la potestad de la voluntad, como la volición, y otra que depende de un acto primero, como sucede en la intelección. Por ello, el alma elige libre y contingentemente en virtud del primero, y procede naturalmente en virtud del segundo ${ }^{29}$. De modo que no solamente no hay bien en los objetos, sino que la acción no se decide a partir de una mirada exterior.

Esto conduce, evidentemente, a comprender los lazos entre la inteligencia y la voluntad de manera muy diferente de la indicada por Santo Tomás. En efecto, Ockham rechaza las distinciones formales que le parece amenazan la unidad del hombre; luego, identifica las facultades con el sujeto concreto que quiere y entiende. No hay lugar para distinguir entre el alma y sus potencias ${ }^{30}$; ni posibilidad de formular una distinción entre los actos de la inteligencia y de la voluntad ${ }^{31}$, respecto de las facultades mismas.

$Y$ aún cuando Ockham afirma la existencia en el hombre de una pluralidad de formas realmente distintas entre sí, no está dispuesto a admitir una distinción real entre sus facultades. Pues así como se negó a admitir que el alma sensitiva posea potencias realmente distintas del alma misma - a menos que por potencias se entienda simplemente las disposiciones accidentales en los diversos órganos de los sentidos-; paralelamente, también se negó a admitir que el alma o forma racional posea facultades realmente distintas de la misma alma racional.

El alma racional es inextensa y espiritual, y no puede tener partes ni facultades ontológicamente distintas. Lo que se llama el entendimiento es simplemente el alma racional que entiende, y lo que llamamos voluntad es simplemente el alma que quiere. El alma racional produce actos; y la potencia o facultad intelectiva «no significa solamente la esencia del alma, sino que connota el acto de entendimiento. Y lo mismo acontece con el caso de la voluntad $»^{32}$..Entendimiento y voluntad son pues, en cuanto términos connotativos; distintos porque un acto de entendimiento es realmente distinto de un acto de querer. Pero si nos referimos a aquello que produce los actos, entendimiento y voluntad no son realmente distintos. $Y$ aquí es el principio de economía el que se aplica para eliminar posibles principios o facultades realmente distintos: hay una sola alma racional que puede producir actos diferentes ${ }^{33}$.

El modelo de esta concepción del hombre sigue siendo Dios: el entendimiento divino es idéntico a la voluntad y a la esencia divina. Y si bien podemos referimos a la voluntad, al entendimiento y a la esencia divina; la realidad a la que se hace referencia es un ser único y simple. Por ello no conviene hablar de ideas divinas como una especie de factor intermediario en

\footnotetext{
29 G. de Ockham, IV Sent, q. 16, (OTH VII, 358-9).
}

30 «Sed distinguo de potentia animae: nam potentia uno modo accipitur pro tota descriptione exprimente quid nominis, alio modo accipitur pro illo quod denominatur ab illo nomine vel conceptu. Primo modo loquendo de intellectu et voluntate, dico quod distinguuntur, nam descriptio exprimens quid nominis intellectus est ista "quod intelectus est substantia animae potens intelligere'. Descriptio voluntatis est quod est 'substantia animae potens velle'. Nunc autem istae descriptiones possunt accipi pro vocibus vel conceptibus vel pro rebus». G. de Ockham, II Sent, q.20 (OTH V, 435).

31 G. de Ockham, II Sent., q. 20 (OTH V, 425-447).

32 G. de Ockham, II Sent., 20 (OTH V, 435)

33 G. de Ockham, II Sent., 20 (OTH V, 436) 
la creación: Dios puede conocer a las criaturas y crearlas sin intervención de ninguna «idea» ${ }^{34}$. Ellas no son, en consecuencia, realidades distintas de la esencia divina, ni tampoco distintas entre sí.

Los términos voluntad, entendimiento y esencia se identifican en un sentido absoluto y son sinónimos: «si un nombre fuera utilizado para significar precisamente la esencia divina y nada más, sin ninguna connotación de otra realidad, y, semejantemente, si un nombre se utilizara para significar de la misma manera la voluntad divina, esos nombres serían simplemente sinónimos; y todo lo que se predicase de uno podría ser predicado de otro $)^{35}$. En consecuencia, si los términos esencia y voluntad se toman en sentido absoluto, podría afirmarse, indiferentemente, que la voluntad divina es la causa de todas las cosas, o que lo es la esencia divina; pues el significado de ambas proposiciones es equivalente. Pero, tanto si hablamos de la esencia divina como si preferimos hablar de la voluntad divina, ha de quedar claro que Dios es la causa inmediata de todo lo creado, aunque esto no pueda ser filosóficamente demostrado ${ }^{36}$.

\section{LA LIBERTAD HUMANA FRENTE A LA LIBERTAD DIVINA}

Como es posible inferir desde los elementos precedentes, la ética ockhamista se ha desarrollado sobre un modo característico de entender la libertad que se aproxima mucho a la sensibilidad de la moderna antropología.

Ockham define la libertad como la ausencia de necesidad que halla su fundamento en una cierta indiferencia, en virtud de la cual la voluntad permanece indeterminada frente a su objeto pudiendo obrar o no, querer una cosa y su contraria. La libertad aparece asi, como ausencia de coerciones, como la simple posibilidad de elección frente a un objeto, significando con ello la capacidad propia del hombre de responder de una manera no predeterminada unívocamente frente a cierta situación.

Libertad, es para Ockham, capacidad de autodeterminación; la sola voluntad concurre como causa para determinar su acción; siendo, por tanto, capaz de actuarse a sí misma. El modelo de la libertad humana es, también en este caso, trabajado desde la libertad divina; el enfoque metodológico es análogo.

La exposición ockhamista utiliza el lenguaje y la doctrina aristotélica del libre arbitrio, pero desde una semántica completamente diversa; pues la libre elección aristotélica se produce a partir de un primer reconocimiento de opciones entre las que el agente elige. Y por ello la libertad aparece como una función del intelecto.

Ockham desestima este elemento fundamental del aristotelismo al invertir la perspectiva del análisis; insiste que la voluntad es un poder activo cuya opción fundamental - actuar o no

34 G. de Ockham, I Sent., 35, 5 (OTH IV, 502 y ss.) La discusión ockhamista de las ideas divinas ilumina tanto la perspectiva medieval general como la propia mentalidad de Ockham. El respeto por san Agustín en la edad Media era demasiado grande para que fuese posible que un teólogo se atreviese a rechazar una de sus teorias principales. En consecuencia, encontramos el lenguaje de la teoría conservado y utilizado por Ockham quien hablaba de ideas distintas, y de esas ideas como modelos o ejemplares de la creación. Por otra parte, valiéndose del principio de economía y decidido a desembarazarse de todo lo que parecía intermediar entre el Creador omnipotente y la criatura, de modo que gobernase la voluntad divina, Ockham recortó de la teoría todo trazo de platonismo e identificó las ideas con las criaturas mismas, en tanto que producibles por Dios y conocidas por Él desde la eternidad como producibles. Abelardo, aun rechazando el ultrarrealismo había conservado la teoría de las ideas universales en Dios, en gran medida por respeto a san Agustín; pero Ockham eliminó esas ideas divinas universales. Su versión de la teoria es consecuente con el principio general de que solamente hay existentes individuales, y con su permanente intención de eliminar los factores de los cuales se pudiera prescindir.

35 G. de Ockham, I Sent., 45 (OTH IV, 664).

36 G. de Ockham, I Sent., 45 (OTH IV. 668). 
actuar - no depende del intelecto que, no actualiza ni determina a la voluntad ${ }^{37}$. De modo que la libertad positiva de la voluntad permanece conectada al alma y sus voliciones; es más es el alma entendiendo y queriendo.

Por ello el análisis lingüístico que desarrolla Ockham sobre la libertad nos la revela asimismo, y enlazando ahora con la metodología propia de todo su discurso filosófico, como un término connotativo que significa a la misma voluntad y a la inteligencia definidas en su propia actividad ${ }^{38}$.

La argumentación del maestro inglés resulta filosóficamente débil: en clave física ha mostrado la dificultad para probar la causalidad actual; por ello tampoco puede probar que el alma produce su efecto de un modo contingente ${ }^{39}$. Sin embargo, el orden de la experiencia le provee la ayuda para una justificación que le satisface: los hombres se experimentan como agentes libres; y por ello la demostración lógica le parece, en este caso, superflua.

La autodeterminación de la voluntad es descrita así: «yo puedo causar algo de un modo no determinado y contingente, en cuanto soy apto para producir o no un mismo efecto ${ }^{40}$. Además, el hecho que censuremos o alabemos a las personas, es decir, que les imputemos responsabilidad por sus acciones, manifiesta que aceptamos la libertad como una realidad: «Ningún acto es culpable a menos que esté en nuestro poder. Nadie culpa a un hombre - ciego de nacimiento- por el hecho de haber nacido ciego, pero si fuese ciego en virtud de un acto propio, entonces sería culpable» ${ }^{41}$.

La voluntad es capaz de producir acciones contrarias o posponer una acción en circunstancias idénticas. La activa operación selectiva de la voluntad libre se magnifica cuando se la compara con el regular y predecible curso de la naturaleza, ámbito propio del determinismo. En el pensamiento de Ockham lo natural y lo libre son nociones antitéticas. La voluntad puede pasar de la potencia al acto por sí misma, y sin ser determinada por una causa en acto. El principio del omne quod movetur sólo es válido en el ámbito de los agentes naturales pero no lo es en sede antropológica. La definición de libertad simplemente reitera la experiencia de la voluntad de ser una causa sui.

Sin embargo parece contrario al principio de contradicción que una facultad pueda producir o no algo, en un determinado instante; pues en ese caso, se verificaría que A es, simultáneamente, B y no B. Ockham explica que las posibilidades deben ser comprendidas disyuntivamente $^{42}$. La voluntad puede elegir o no su efecto, pero no de un modo simultáneo. La

37 «Loquendo de primo actu, posito omni sufficienti et necessario requisito ad talem actum, puta, ad actum voluntatis; si objectum cognoscatur et Deus velit concurrere cum voluntate ad causandum quando placet voluntati, potest voluntas ex sua libertate sine omni alia determinatione actuali vel habituali actum illum et eius objectum elicere vel non elicere. Et ideo respectu illius actus non oportet in aliquo quod determinetur voluntas nisi a seipsa» G. de Ockham, IV Sent., q. 16, (OTH VII, 359). Podrá consultarse paralelamente: G. de Ockham, I Sent., d. 38 q. 1 (OTH IV, 574 y ss.) y Quodl., I, q. 16 (OTH IX, 87). Ockham puntualiza que una pasiva indiferencia con relación a los opuestos no puede justificar el concepto de libertad: « Si (voluntas) esset potentia passiva et nullo modo activa, non videtur quomodo possit salvari eius libertas» G. de Ockham, IV Sent., q. 16 (OTH VII, 358)

38 «Imaginantur enim ac si libertas esset unum aliquid reale distinctum aliquo modo ex natura rei a voluntate vel non omnino idem cum voluntate; quod tamen non est verum. Sed est unum nomen connotativum importans ipsam voluntatem vel naturam intellectualem connotando aliquid contingenter posse fieri ab eadem» $G$ de Ockham, I Sent., d. 10, q. 2, (OTH, III, 344)

39 «Circa secundum sunt duae difficultates. Prima est utrum possit probari sufficienter qud voluntas sit libera. Secunda supposita libertate voluntatis, utrum possit probari quod voluntas sit potentia activa. Circa primum dubium, dico quod non potest probari per aliquam rationem quia omnis ratio probans accipit aeque dubia et aeque ignotum conclusioni vel ignotius» G. de Ockham, Quod. I, q. 16 (OTH IX, 87-88)

40 G. de Ockham, Quod., I, q. 16 (OTH IX, 87)

41 G de Ockham, III Sent., q. 10 y 11 . (OTH VI, 315 y ss.)

42 Cfr. A. Garvens, «Die Grundlagen der EthikWilhelms von Ockham», Franziskanische Studien, 1934, (21), 249-262 y K. Mc.Donnel, «Does William of Ockham Have a Theory of Natural Law?», Franciscan Studies, 1974, (34), 383-392. 
explicación requiere un análisis temporal de la libertad que tiene importante consecuencias para la teología ockhamista en tanto esta división es en principio, la misma para la voluntad humana y para la libertad divina. Divide el proceso de causar libremente en tres momentos: «antes, durante y después de». Antes de la elección, la voluntad está indeterminada para producir o no, un acto. En el momento de elegir, la voluntad está en sí misma determinada y es incapaz de producir actos contrarios simultáneamente. Finalmente, después de elegir su acto, la voluntad humana recupera su indiferencia para continuar o terminar su decisión. Y en este itinerario la única causa de la acción contingente es la preferencia personal. La prueba de la libertad humana se alcanza así en estas tres etapas y debe sostener un perfil contingente e indiferente antes y después de cualquier elección definida. En este discurso ockhamista no late el ser, sino el tiempo, y particularmente el tiempo del discurso, el antes y el después de proposiciones que se verifican a través de las intuiciones sucesivas de un proceso.

Ockham quiere señalar con ello que mientras la elección no ha sido efectuada, la voluntad permanece esencialmente indeterminada a querer esto antes que aquello. La libertad no es sino una con la espontaneidad del querer que sigue siendo el único elemento posible de contingencia ante las determinaciones de la razón. Siguiendo una vía voluntarista se ha colocado la indeterminación del querer en el origen del libre albedrío.

Aristóteles ha enseñado, por el contrario, que todo acto de elección involucra la felicidad en cuanto fin necesario al cual tiende la naturaleza humana. Por ello algunos escolásticos Gil de Roma, en particular, como interlocutor inmediato de Ockham ${ }^{43}$ - infieren que la voluntad es un poder pasivo y que la libertad humana está limitada a un fin que es necesario. De allí que su reflexión sobre la libertad se extendiera al problema de la relación entre la voluntad y su adhesión - necesaria o libre- al fin último; cuestionándose si la voluntad permanece indeterminada frente al fin, conocido en la intuición perfecta propia de la visión beatífica.

La respuesta de Santo Tomás y de Duns Escoto — sobre quien se apoya Ockham- fue diversa. Para Santo Tomás el hombre es libre en la elección de los medios que lo conducen al fin último; pero no es libre frente a él cuando lo conoce adecuadamente en sí y de modo directo; la voluntad es libre sólo cuando los dictámenes de la razón en orden del fín último no se imponen con la fuerza de la verdad absolutamente evidente ${ }^{44}$. Para Escoto, en cambio, la voluntad debería siempre estar en grado de sustraerse a las solicitaciones del intelecto aún frente al bien absoluto ${ }^{45}$.

Ockham no ha enfrentado sistemáticamente este problema, sin embargo su pensamiento surge de un número relevante de afirmaciones que se encuentran dispersas en su obra ${ }^{46}$.

Su punto de partida ya es singular: señala que no puede ser probado filosóficamente que el goce de la esencia divina nos sea posible. E incluso si bien por la fe sabemos que es posible, podemos quererlo o no a este fin último. Más todavía, no queremos necesariamente ni siquiera la felicidad perfecta en general porque el entendimiento puede considerar que la felici-

43. Ockham singularizó a Gil de Roma (1247-1316) como uno de estos escoláticos que consideró a la voluntad como un poder pasivo. Se puede revisar el análisis ockhamista de la doctrina de Gil de Roma y su refutación en: Expositio Physicorum, II, texto 48 (OPH IV, 318 y ss.) y Quaestiones Physicorum, q. 127 y 128 (OPH VI, 738744).

44 Tomás de Aquino, STh. I, 82, 1

45 «Voluntas sic determinatur ad volendum beatitudinem et ad nolendum miseriam, quod si eliciat aliquem actum circa ista obiecta, necessario et determinatum elicit actum volendi respectu beatituninis, et nolendi respectu miseriae, non tamen absolute determinatur ad unum actum eliciendum vel aliumi Ordinatio Scoti, IV, dist. 49, q. 10 ; ed. Vivès, XXI, 333.

46 A. Garvens, «Die Grundlagen der Ethik Wilhelms von Ockham», Franziskanische Studien., 1934, (21), 243-273; 360-408. E. Bettoni, $1 /$ problema del fine ultimo nel pensiero di Ockam, Studia mediaevalia et mariologica P. Corolo Balic OFM septuagesimum explenti annum dicata, Romae, 1971, 227-247. Otros trabajos: I Boh, «An examination of Ockham's aretetic logic», Archiv für Geschichte der Philosophie, 1963, (45), 259-268; J. Miethke, Ockhams Weg zur Sozialphilosophie, Berlin, 1969, 300-347. 
dad perfecta no es posible y que la única condición posible en nuestras vidas es ésta en la que nos encontramos ${ }^{47}$.

En suma: no hay argumentos válidos que demuestren que existe un bien infinito; y si bien la voluntad tiene un fin último, no es posible establecer racionalmente que ese fin último sea Dios. Puede ser la felicidad en general frente a la cual la voluntad humana permanece libre; puede ser también un bien finito aquel que sacie la sed de felicidad propia del hombre; quedando también en manos del hombre la activa renuncia a la felicidad ${ }^{48}$.

De modo que la filosofía del maestro inglés no está en condiciones teóricas de demostrar que el fin del hombre consiste en la visión beatífica. Todo lo que sabemos con certeza sobre la existencia y sobre la naturaleza del fin último, y sobre el acto con el cual la voluntad lo posee, lo enseña la revelación ${ }^{49}$.

Por último, partiendo de la distinción escolástica entre fin último de derecho y fin último de hecho, y en constante referencia a la experiencia, Ockham concluye que si existen hombres que no tienden al fin último de derecho es porque la voluntad puede orientarse hacia un fin último que en realidad no es propiamente el último fin y frente a él, la voluntad permanece libre. $Y$ agrega que a menos que la voluntad sea puesta en una situación imprevisible por Dios, puede siempre rechazar abrazarse al último fin en cualquier modo que le venga presentado ${ }^{50}$, aún intuitivamente. Pues la voluntad siempre permanece indeterminada y por lo tanto en grado de autodeterminarse frente a cualquier objeto, dado que cualquiera sea la fuerza persuasiva del entendimiento, éste nunca determina a la voluntad.

Si bien la tesis ockhamista puede parecer como una toma de posición nueva que se destaca del pensamiento de los escolásticos, entiendo que Ockham sólo profundiza el camino que ya habían iniciado San Buenaventura y Duns Escoto cuando afirmaron la insuficiencia de una fundamentación filosófica de la ética. Ockham, por su parte, expone tres criterios - dos históricos y uno metodológico- para rechazar una posible armonización:

1. En primer término, Aristóteles y sus comentadores no lograron afirmar cuál es el fin último del hombre; por lo tanto no es verosímil que tal fin pueda ser descubierto por la indagación filosófica.

2. Además, los argumentos de los más autorizados maestros de la escolástica del siglo XIII que han creído demostrar que Dios es el fin último, son débiles, pues tienen carácter persuasivo pero no apodíctico. Ockham no nos dice que Dios no sea el fin último del hombre; afirma simplemente que la razón humana no está en grado de establecer con exactitud cuál es el fin último de la creatura libre. No hay personalmente para Ockham un conflicto entre la verdad alcanzada por la razón y aquella de la fe, pero sí entendemos que lo ha gestado para el pensamiento posterior.

3. Y, finalmente, la clásica aplicación del principio de economía al ámbito teológico: si es una verdad de fe que Dios es el fin último del hombre, no es conveniente que pueda devenir objeto de una demostración ${ }^{51}$.

Al haber suprimido toda fundamentación metafísica del orden moral, la doctrina ockhamista de la libertad tiene un marcado tono autoritario. Una voluntad libre está sujeta a obliga-

\footnotetext{
47 G de Ockham, I Sent., 1, q. 6 (OTH I, 486 y ss.)
}

48 «Voluntas contingenter et libere $\rightarrow$ modo exposito - fruitur fine ultimo ostenso in universali, quia scilicet diligere beatitudinem potest et non diligere, et potest appetere sibi beatidudinem et non appetere» $G$ de Ockham, I Sent., 1, 6 (OTH. 503).

49 D. Clark, «Ockham on Human and Divine Freedom», Franciscan Studies, 1978, (16), 154.

50 «Dico quod finem ultimum, sive ostendatur in generali sive in particulari, sive in via in patria, potest absolute voluntas eum velle vel non velle vel nolle» G de Ockham, I Sent, 1, 6 (OTH I, 506). Cfr. Asimismo, G. de Ockham, IV Sent, 16 (OTH VII, 350 y ss.)

51 Cfr. D. Clark, «Ockham on Human and Divine Freedom», 144-148. 
ción moral; y en punto a ello el hombre está moralmente obligado a querer lo que Dios le ordena querer y a no querer lo que Dios le ordena no querer. El fundamento ontológico del orden moral radica en la dependencia en que el hombre está respecto a Dios, como criatura que es, respecto de su Creador. $\mathrm{Y}$ el contenido de la ley moral lo proporciona el precepto divino: «EI mal no es otra cosa que hacer algo cuando se está bajo la obligación de hacer lo opuesto» ${ }^{52}$.

Una vez más, esta personal concepción de la ley moral está intimamente relacionada con la insistencia de Ockham en la omnipotencia y libertad divinas. Cuando esas verdades se aceptan como verdades reveladas todo el orden creado, incluida la ley moral es vista como contingente en el sentido de que no sólo su existencia sino también su esencia y carácter dependen de la voluntad creadora y omnipotente de Dios.

Pero la obligación que es el resultado de la imposición divina no puede afectar a Dios mismo: «Por el hecho mismo de que Dios quiere algo, es bueno que ese algo se haga (...). Por lo tanto, si Dios causase el odio a Él, en la voluntad de alguien, es decir, si Dios fuese la causa total de ese acto, ni pecaría aquel hombre ni pecaría Dios, porque Dios no está bajo obligación alguna y el hombre no estaría [en tal caso] obligado porque el acto no está bajo su propio poder ${ }^{53}$. Dios puede hacer todo y ordenar todo lo que no implique contradicción lógica. Huelga decir que Ockham no trataba de sugerir que el odio a Dios sea un acto legítimo y menos aún animar a que estos actos se cometiesen. Su tesis era que tales actos son indebidos sólo porque Dios los ha prohibido. Nuevamente, su intención es subrayar la omnipotencia y libertad divinas, afirmando que no tiene sentido alguno que se busque para la ley moral una razón ulterior al fiat divino.

\section{NUESTRO BALANCE Y EVALUACIÓN}

Varios son los elementos que se desprenden de la doctrina ockhamista de la libertad:

1. En primer término, la ruptura con el principio aristotélico del omnia quod movetur $a b$ alio movetur $^{54}$. Sin un agente externo no hay razón para explicar la transferencia de la inacción a la acción. Ockham describe la libertad como la capacidad para producir un acto sin una determinación precedente. La experiencia de la libertad nos la muestra como un poder espontáneamente activo, una suerte de motor inmóvil. En suma, la libertad de la voluntad prueba que para ser en acto, no es necesario ser movido por otro.

2. La teoría de la libertad de Ockham se mueve en un ámbito voluntarista en el que se configura una completa equiparación entre libertas y voluntas. La libertad no es un atributo de la voluntad, sino que es un acto que se pone a sí mismo como libre. Sin dudas la espontaneidad del querer - tan fuertemente afirmada por San Anselmo- alcanza en Ockham una expresión definitiva. Yendo aún más allá del dinamismo de Aristóteles, opone radicalmente el orden de las naturalezas, que es el de la necesidad, al orden de las voluntades, que es el de la libertad. Toda naturaleza es esencialmente determinada; la voluntad, en cambio, es un principio de autodeterminación. Por lo demás, en la indeterminación de la voluntad no debe verse la señal de una insuficiencia o de una imperfección, pues testifica la excelencia de una facultad no determinada por un acto antecedente. En efecto, Ockham adjudica la determinación al ámbito del conocimiento; y la libertad al ámbito respectivo de la voluntad. La contingencia de la elec-

52 G. de Ockham, IV Sent., q. 11, (OTH VII, 196-7).

53 G de Ockham, IV Sent., q.11, (OTH VII, 198).

54 El principio es aristotélico (Cfr. Aristóteles, Physica, VII, cap. 1241 b 25-242a 16). Es parcialmente rechazado por Duns Escoto en virtud de la evidencia del automovimiento de la voluntad: Roy Effler, John Duns Scotus and the Principle «Omne quod movetur ab alio movetur», Franciscan Institute, St. Bonaventure, 1962. 
ción no se debe a los juicios racionales que proponen la alternativa entre actos posibles, sino a la espontaneidad del querer. La libertad se concentra, pues, en la indeterminación radical de la voluntad, cuyas decisiones imprevisibles surgen desde ella como de una fuente o principio no determinado ${ }^{55}$.

3. En cuanto al fin último: Ockham sostiene que la naturaleza carece de una orientación específica; no está abierta, ni determinada por la verdad ni por el bien. Sólo el agente libre y consciente actúa en vista de un fin que él mismo se impone mientras que el universo físico es predecible y perfectamente determinado. El hombre, que no es una realidad enteramente natural, puede ser causa sui, y por ello, introducir en el mundo posibles comienzos de nuevas causaciones. La libertad aparece como un comienzo; mientras que en la naturaleza no hay verdaderos inicios, sino determinación y continuidad. Ockham ha configurado el dilema moderno de la libertad versus el determinismo.

4. La antropología sobre la cual Ockham trabaja el tema de la libertad concibe el hombre como imagen de un Dios todopoderoso, autosuficiente y causa de sí: en Dios el conocimiento es una y la misma cosa que su esencia. En verdad, la multiplicidad de los nombres divinos se encuentra sólo en nuestros signos. Aplicando este criterio, Ockham unifica y construye el intelecto del hombre sobre la voluntad y ella sobre el modelo mismo de la omnipotencia divina.

5. Desde la doctrina de la libertad de Ockham es imposible construir una teoría de la naturalidad de la ley moral. Aquino y Escoto vinculan la elección humana de la bondad con el Infinito Bien. Ockham no. Ockham sugiere que la libertad es la característica de la naturaleza humana ${ }^{56}$; la personal creatividad con la que Pico eleva su Oración sobre la Dignidad del Hombre es lógicamente compatible con esta teoría ockhamista de la libertad aún cuando las actitudes de Ockham continúen siendo las de un pensador medieval.

6. Ockham sitúa, de este modo, dos voluntades enfrentadas. Sin duda en el sistema ockhamista se supera el enfrentamiento pues la voluntad de uno de los extremos en un momento acaba siendo expresión de la voluntad del otro, en ese mismo momento; pues la omnipotencia y la conservación garantizan la continuidad a estos actos. Pero, sin duda, inicia una perspectiva en la que despunta un conflicto. En efecto: podría suceder que la exaltación de la omnipotencia divina se imponga a la libertad del hombre; o bien, que, contrariamente, sea el hombre quien fije con total autonomía su destino moral; o finalmente, que la lucha se sostenga de manera indefinida; posiciones todas por las cuales transita el pensamiento posterior. Le corresponde a Ockham haber separado la voluntad humana de la naturaleza, instaurando, con ello las bases del conflicto moderno.

\author{
Olga L. Larre \\ Consejo Nacional de Investigaciones Científicas y Técnicas (CONICET) \\ República Argentina \\ C. E.olgalarre@comnet.com.ar
}

55 Cfr. J. Carreras y Artau, Ensayo sobre el voluntarismo de J. Duns Scot, Gerona, 1923, 85-90.

56 «Secundo sic: creatura rationalis est perfectior omni creatura irrationali, ergo illud accidens per quod distinguitur maxime a creatura irrationali erit perfectius; sed videtur quod creatura rationalis magis distinguitur per volitionem quam per quamcumque delectationem. Assumptum patet quia maxime distinguitur per libertatem quae est principium volitionis») G de Ockham, I Sent., d. I, q. 3 (OTH: I, 426). 